\title{
Understanding the ellagitannin extraction process from oak wood
}

\author{
I. García-Estévez ${ }^{\text {a }}$, C. Alcalde-Eon ${ }^{\text {a }}$, L. Le Grottaglie ${ }^{\text {b }}$, J.C. Rivas-Gonzalo ${ }^{\text {a }}$, \\ M.T. Escribano-Bailón ${ }^{\text {a,* }}$ \\ ${ }^{a}$ Grupo de Investigación en Polifenoles (GIP), Unidad de Nutrición y Bromatología, Facultad de Farmacia, Universidad de Salamanca, Salamanca, Spain \\ ${ }^{\mathrm{b}}$ University of Naples Federico II, Department of Chemical Sciences, Via Universitá, 100 Portici, 80055 Naples (NA), Italy
}

\section{A R T I C L E I N F O}

\section{Article history:}

Received 6 June 2014

Received in revised form 1 October 2014

Accepted 20 October 2014

Available online 24 October 2014

\section{Keywords:}

Oak ellagitannins

Extractability

Kinetic model

Oak chips

Toasting

Model wine solution

\begin{abstract}
A B S T R A C T
The extractability of the main oak ellagitannins has been studied in five model solutions containing different types of oak chips (two sizes and different toasting degrees for each size). A new extraction kinetic model has been proposed from the quantitative experimental results obtained by means of HPLC-ESI-MS/MS-multiple reaction monitoring method. The model considers an initial extraction (i.e., washing step) followed by a diffusion step, which involves two different processes that follow first-order kinetics at different rates. Differences in the extractability of the ellagitannins in the different model solutions have been observed and explained on the basis of the kinetic model here proposed.
\end{abstract}

(C) 2014 Elsevier Ltd. All rights reserved.

\section{Introduction}

Aging red wines in oak wood barrels is an enological practice aiming at improving their overall quality. Interactions of wood and air with wine play an important role in increasing its complexity. ${ }^{1,2}$ First, regarding wine-air interactions, the structure of the barrel allows a controlled entrance of oxygen, which is essential to the polymerization reactions between different types of flavonoids, leading to a modification of the organoleptic properties of the wine. Furthermore, wood can affect wine composition and, consequently, organoleptic properties through different mechanisms. On the one hand, wine compounds can be adsorbed onto wood surface. On the other hand, different types of compounds, such as ellagitannins, can be extracted from wood to the wine due to the hydro-alcoholic nature of the latter. Ellagitannins can take part in oxidation reactions $^{3}$ that may favor the polymerization reactions between flavanols and between flavanols and anthocyanins. Furthermore, they can directly react with these types of compounds giving rise to flavano-ellagitannins or anthocyano-ellagitannins. ${ }^{4-6}$ As a consequence, ellagitannins can modulate wine astringency and color through interactions with these compounds. These compounds can also contribute directly to the astringent mouthfeel of the oakmatured red wines ${ }^{7}$ since they are able to interact with hydrophobic constituents of the oral cavity. ${ }^{8}$ Furthermore, Michel and

\footnotetext{
* Corresponding author. E-mail address: escriban@usal.es (M.T. Escribano-Bailón).
}

co-workers ${ }^{9}$ have reported that the levels of ellagitannins in wine also affect the roundness and amplitude of the red wine.

The amount of ellagitannins released into the wine depends on the content in the oak wood barrel, which in turn is dependent on several factors. First, the botanical origin of the oak that conditions the initial levels of the wood employed in cooperage: the highest levels of ellagitannins have been observed for Quercus robur $\mathrm{L}$. followed by Quercus petraea (Matt.) Liebl and with the lowest levels being observed for the American species (Quercus alba L.). ${ }^{10-14}$ Furthermore, other factors as the age and geographical origin of the tree as well as the length and type of seasoning and toasting of the wood, condition the levels of ellagitannins in the oak barrels. ${ }^{11,14-18}$ Respecting toasting, it has been reported that it can produce a significant degradation of ellagitannins, ${ }^{14,16,18,19}$ which is also modulated by the type of seasoning previously carried out ${ }^{15}$ and the use of water during toasting. ${ }^{16}$ Furthermore, toasting does not only affect ellagitannin content in the wood but also the extractability of these compounds from wood to wine. Toasting can cause the breakdown of cellular structures, ${ }^{15}$ which might favor the extraction of these compounds from the wood to the wine.

The main ellagitannins in oak wood are castalagin and vescalagin, which can represent up to $60 \%$ of the total soluble ellagitannin content. ${ }^{12,17}$ Lyxose and xylose derivatives (grandinin and roburin $\mathrm{E}$, respectively) as well as dimeric forms (roburins $\mathrm{A}, \mathrm{B}, \mathrm{C}$, and D) are also present in oak wood. ${ }^{11,12,20,21}$ Despite their related structures, the reactivity and biological properties are different from one oak wood ellagitannin to another. Between vescalagin 
and castalagin, the only structural difference lays on the orientation of the hydroxyl group at $\mathrm{C} 1$ of the glucose moiety. However, it has been reported that vescalagin but not castalagin can take part in several nucleophilic reactions leading to the formation of flavano-ellagitannin (accutisimins) compounds or anthocyanoellagitannin pigments, which has to be related to the orientation of this hydroxyl group. ${ }^{21,22}$ In addition, it has also been reported that as a consequence of the different conformation in $\mathrm{C} 1$ of the glucose molecule, vescalagin and castalagin express drastic differences in biological activity.

However, the content of ellagitannins in wine depends not only on the content in oak wood but also in their extractability. The amount extracted from oak wood can be influenced by the degree of toasting of the wood or by the type of aging performed. As previously indicated, although toasting decreases the levels of the ellagitannins in the wood by thermal degradation ${ }^{14,16,18,19}$ it can favor the release of these compounds to the wine because of the cellular breakdown caused by toasting. ${ }^{15}$ On the other hand, the extraction of the ellagitannins takes place differently if the aging is performed in oak barrels or with lower-cost alternative aging systems, such as chips. ${ }^{24}$ It has been reported that the ellagitannin maximum concentration is lower but is reached earlier in wines aged in stainless steel tanks with oak chips than in the same wines aged in oak barrels. ${ }^{24}$ Nevertheless, the studies on ellagitannin extractability are scarce. First studies on ellagitannins extraction were carried out in model casks ${ }^{25}$ using a $12 \%$ ethanol-model solution. However, the results of that study do not strictly provide information on how the extraction process occurs since, due to the length of the study (200 days), other processes such as oxidation or polymerization have probably influenced the concentration determined at the end of the study. Karvela and co-workers ${ }^{26}$ studied the extractability of ellagitannins from non-toasted oak chips $(Q$. petraea) in different model systems observing that the extraction followed second-order kinetics. However, this extractability did not correspond only to ellagitannins but also to gallic and ellagic acids, since it was calculated from the total polyphenols content. Chira and co-workers ${ }^{19}$ have studied the evolution of the total ellagitannin content in wines aged in stainless steel tanks with seven different types of winewood, observing above all quantitative differences between samples. Jourdes and co-workers, ${ }^{24}$ on the contrary, studied the individual evolution of the main $C$-glucosidic ellagitannins in red wines aged in oak barrels and in stainless steel tanks with oak chips. However, no kinetic models were proposed in none of these two studies. ${ }^{19,24}$ Taking into account the different chemical reactivity and biological activity shown by the different ellagitannins, it seems relevant to determine the individual extractability of the main oak ellagitannins. Thus, the objective of the present study is to define a kinetic model that would allow the understanding of the ellagitannin extraction process from oak wood. Furthermore, once the kinetic model defined it will be employed to establish comparisons among the individual extractability of the main oak ellagitannins and to evaluate how is the extractability affected by the toasting degree and by the size of the wooden chips.

\section{Results and discussion}

\subsection{Selection of kinetic model}

Several mathematical models have been described in literature to explain the kinetics of solid-to-liquid extractions. Some of them (Table 1) have been used to describe the extraction of phenolic compounds from different plant sources, ${ }^{26-36}$ and they have been tested in the present study to explain the extraction of the individual ellagitannins from oak chips with different characteristics. Table 1 shows the results obtained from fitting the data of the extraction of castalagin (the main oak ellagitannin) from the biggest light-toasted oak chips (8L). The usefulness of the different kinetic models was assessed by the values of $R_{\text {adj }}^{2}$ and RMSE.

The kinetic models 1-3 (Table 1 ) have previously been used to explain the kinetics of extractions by considering one only step of extraction. For example, the basic non-linear kinetic model (model 1) has previously been employed to describe the kinetics of polyphenol diffusion from oak chips into model matrices, ${ }^{26}$ whereas Weibull-type distribution (model 2) has been used in literature to explain the extraction of phenolic from different sources. ${ }^{28,29}$ In the present study, however, these models did not provide the best results, mainly due to the fact that they consider the extraction happening in one continuous step. Data fitting using any of these models showed a positive serial correlation in residuals versus time plot at the end of the study. This indicates that these models could not be used to explain the extraction at the latest steps, since they do not consider the depletion of the outermost layers of wood of oak chips and that as a consequence, solvent has to penetrate into the deepest layers to extract the ellagitannins, which implies a decrease of extraction rate.

The worst $R_{\text {adj }}^{2}$ and RMSE values were obtained when basic firstorder equation (model 3, Table 1) was used to try to explain ellagitannins extraction data. Furthermore, it was possible to observe a sinusoidal behavior of residual values (data not shown). Karacabey and co-workers ${ }^{30}$ also obtained a poor goodness of fit when they proposed this model to explain the extraction of resveratrol and viniferin from grape cane. This method considers that extraction takes place at a constant rate, which seems not to be a valid assumption for solid-to-liquid extractions from plant sources.

Solid-to-liquid extraction can also be explained by defining two different steps in kinetic models 4-6 (Table 1). However, although data fitting by using these models showed good $R_{\text {adj }}^{2}$ and RMSE values, as will be seen below, they were still insufficient to explain the ellagitannin extraction from different kinds of oak chips. Peleg's model (model 4) is based on sorption/desorption curves and it describes the extraction in two stages: first-order at the very beginning of the extraction, and zero-order in the latest phase of the process. $^{29-32}$ Likewise, Two-sites kinetic model (model 5) also considers two steps of extraction, although in this case, both steps are first-order kinetics at two different rates, taking into account that extraction of compounds from surface can be much faster than internal diffusion and, as a result, the extraction is limited by internal diffusion. ${ }^{33,34}$ The results of data fitting using any of these

Table 1

Kinetic models used to explain the extraction of ellagitannins from oak chips

\begin{tabular}{|c|c|c|c|c|c|}
\hline & Kinetic model & Equation & $R_{\mathrm{adj}}^{2}{ }^{\mathrm{a}}$ & RMSE $^{a}$ & References \\
\hline (1) & Non-linear & $C=a t^{n}$ & 0.988 & 0.32 & 26 \\
\hline (2) & Weilbull-type & $C=C_{0} \mathrm{e}^{-k t n}$ & 0.984 & 0.29 & 28,29 \\
\hline (3) & First order & $C=C_{\mathrm{eq}}\left[1-\mathrm{e}^{-k t}\right]$ & 0.900 & 1.06 & 30 \\
\hline (4) & Peleg's model & $C=\frac{t}{K_{1}+K_{2} t}$ & 0.941 & 0.69 & 31,32 \\
\hline (5) & Two-sites & $C=C_{\mathrm{eq}}^{\Lambda_{1} \Lambda_{2} t}\left[1-\left[F e^{-k t}\right]-\left[(1-F) e^{-k t}\right]\right]$ & 0.977 & 0.22 & 33,34 \\
\hline (6) & Minchev and Minkov & $C=A-B \mathrm{e}^{-k t}$ & 0.981 & 0.31 & 35,36 \\
\hline
\end{tabular}

a These values were obtained from fitting the data of the extraction of castalagin from the biggest light-toasted oak chips (8L). 
two models showed values of residuals too high at the very beginning. This could be explained because the initial extraction (the extraction at the moment of the addition of the oak chips to the model systems) is not being taken into account in the equations, which represents the main drawback of using these two models to explain ellagitannins extraction from oak chips. On the other hand, Minchev-Minkov's model (model 6, Table 1) describes an initial extraction followed by a diffusion step of compounds from the solid phase. ${ }^{35,36}$ However, the diffusion step is considered as a homogeneous step, and for this reason, this model showed a positive serial correlation in residuals versus time plot at the end of the study, in addition to high values of residuals at the first steps.

Finally, data were fitted by using a new equation here proposed for the first time, named Washing E Two-rates kinetic model (Eq. 1):

$C=C_{\mathrm{eq}}\left[F_{\mathrm{w}}+F_{\mathrm{dij}}\left[1-\left[F e^{-k_{1} t}\right]-\left[(1-F) e^{k_{2} t}\right]\right]\right.$

This model provided the best $R_{\text {adj }}^{2}$ (0.994) and RMSE (0.16) values, thus showing a better fit to extraction data. The Washing $\mathcal{E}$ Two-rates kinetic model (Eq. 1) considers an initial extraction followed by a diffusion step, which involves two different processes that follow first-order kinetics at different rates. With this model it was possible to explain all the steps involved in ellagitannin extraction from oak chips as can be inferred by the good values of $R_{\mathrm{adj}}^{2}$ and RMSE obtained and by the random distribution observed in residual versus time plot.

\subsection{Kinetics of ellagitannin extraction}

Kinetics of the individual ellagitannins extraction from the different kinds of oak chips was explained by using the aforementioned Washing \& Two-rates kinetic model (Eq. 1). The inert conditions employed, together with the short length of the time period (four days) chosen to study the extractability of the ellagitannins, ensured that the extraction was the only process taking place. The model defines a final concentration, $C_{\mathrm{eq}}(\mathrm{mg} / \mathrm{L})$, which corresponds to the maximum concentration of ellagitannin extracted from oak wood. Fig. 1 shows, as an example, the curves of extraction of castalagin from the different kinds of oak chips assayed. The curves of extraction of grandinin, vescalagin, and roburin E are included as Supplementary data (see Figs. S1-S3 in Supplementary data, respectively).

In these curves it can be observed that ellagitannins are released from wood to model wine solution in two main steps, which were considered in the model equation as follows: $F_{\mathrm{w}}$ is the fraction of ellagitannins extracted in the first step, which corresponds to an initial extraction from the surface of the oak chips, i.e., a washing step that takes place immediately after contact with solvent, during the moistening of the oak chips. $F_{\text {dif }}$ is the fraction of ellagitannins that diffuses from the oak chips, in a second extraction step. This second extraction follows first-order kinetics but it could be subdivided in turn in two steps that differ on their rates. $F$ is the fraction of ellagitannins extracted at the fastest rate $\left(k_{1}, \min ^{-1}\right)$,
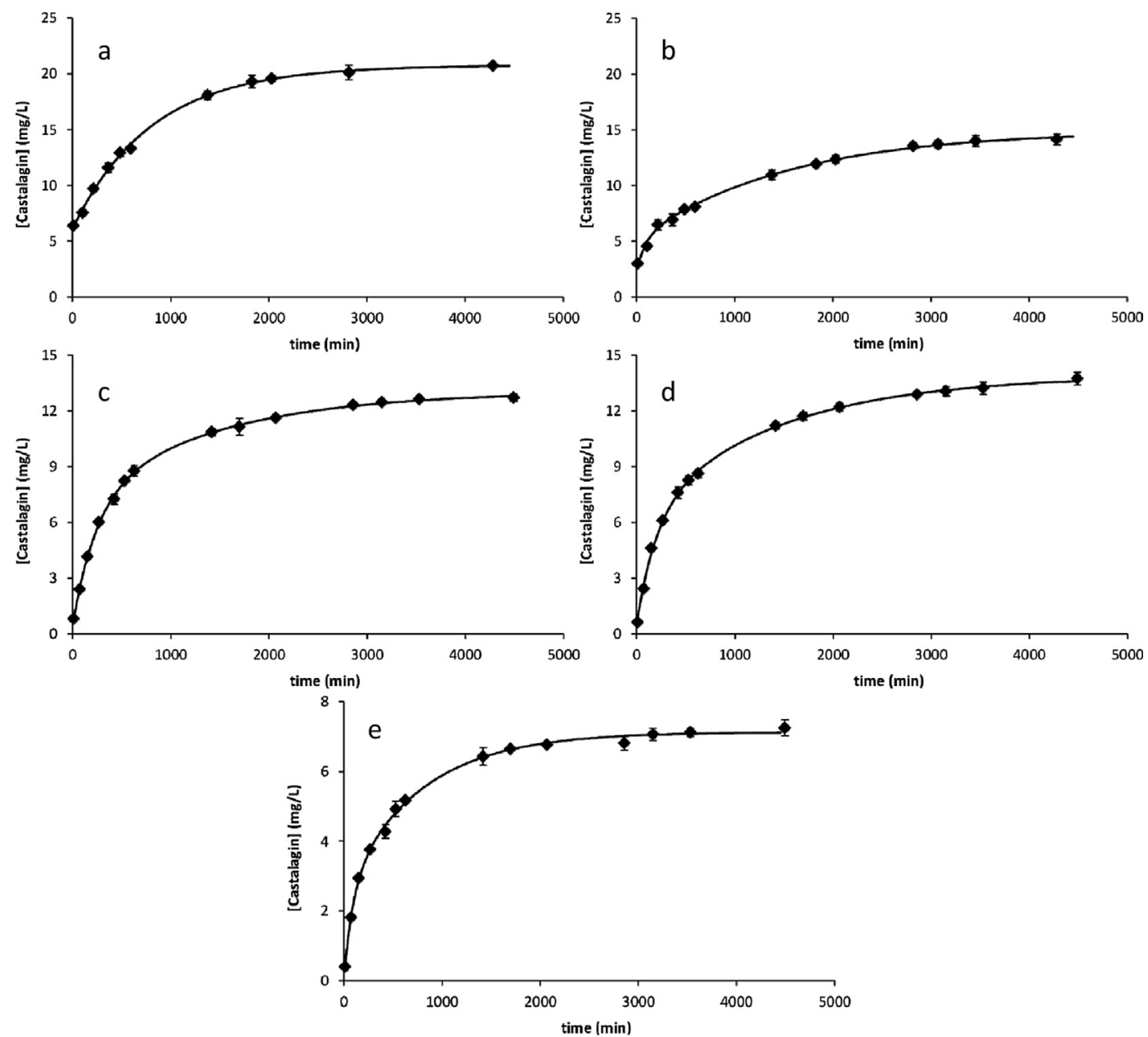

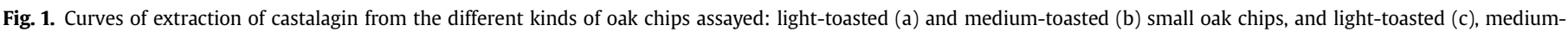
toasted (d), and high-toasted (e) big oak chips. See Section 4 for further details about chips features and sampling procedure. 
which corresponds to the ellagitannins from the outermost layers of the oak chips. The second step of this diffusion process corresponds to the ellagitannin extraction from the inner layers of the oak chips. Hence, the solvent must penetrate into the oak chip and consequently this step takes place at a slower rate $\left(k_{2}, \min ^{-1}\right)$. Table 2 shows the calculated parameters $\left(C_{\mathrm{eq}}, F_{\mathrm{w}}, F_{\mathrm{dif}}, F, k_{1}\right.$, and $\left.k_{2}\right)$, as well as the parameters indicating the goodness of fit $\left(R_{\mathrm{adj}}^{2}\right.$ and RMSE) of the different model systems, for castalagin, vescalagin, grandinin, and roburin E. As can be observed, in all cases high $R_{\text {adj }}^{2}$ and low RMSE values were obtained, thus corroborating the usefulness of this equation to explain the extraction of ellagitannins from oak chips.

2.2.1. Differences on the extractability of the individual ellagitannins. Comparison between the individual ellagitannins was made from the results obtained for light-toasted oak chips (2L and $8 \mathrm{~L}$ ) since these are the cases in which the composition and structure of oak wood are less affected. ${ }^{15}$ As could be expected from the levels of the individual ellagitannins in oak wood, ${ }^{12,17}$ castalagin and vescalagin showed the highest values of $C_{\mathrm{eq}}$, whereas grandinin and mainly roburin E showed the lowest ones (Table 2). Moreover, differences on the fraction of each ellagitannin that is extracted in each process described by the model were observed. The value of $F_{\mathrm{W}}$ is higher for vescalagin than for castalagin, and much higher than for grandinin and roburin $\mathrm{E}$, which means that vescalagin is released slightly easier from oak chips during washing step than the rest of main oak ellagitannins. This difference might be explained, in the case of castalagin, by the differences on the orientation of the hydroxyl group at $\mathrm{C}-1$ of the glucose moiety: vescalagin possesses an exo-positioning $\beta-\mathrm{OH}^{21}$ that may allow the vescalagin to establish hydrogen bonds with the solvent. Thus, during washing step, the release of vescalagin may be favored in relation to castalagin, in which the hydroxyl group $(\alpha-\mathrm{OH})$ is endo-located and may participate in intramolecular hydrogen bonds. ${ }^{21}$ The low values of $F_{\mathrm{w}}$ for grandinin and roburin $\mathrm{E}$ might be related to the more complex structures and to the higher molecular weight of these pentosylated monomers, which may difficult their extraction.

As explained above, diffusion of ellagitannins from oak chips follows first-order kinetics differentiating two steps according to their rates. The fastest step of the diffusion process takes place at a rate $\left(k_{1}\right)$ much faster than the second step $\left(k_{2}\right)$. Depending on the ellagitannin, $k_{1}$ can be from twice to 20 times faster than $k_{2}$. In the same way, the fraction of ellagitannins extracted in each step ( $F$ and $1-F$ ) differs among the different ellagitannins. Castalagin and vescalagin showed extraction rates very similar in both steps but the fraction of castalagin extracted in the first step is higher than that corresponding to vescalagin (Table 2). It might be explained by the lower amounts of vescalagin in the outermost layers of the oak chips as a consequence of the higher proportion of vescalagin released during the washing step. Moreover, grandinin and roburin E showed an $F$ value much lower than those of the other ellagitannins, together with lower rates of extraction for the second step of the diffusion process $\left(k_{2}\right)$, mainly in the case of extraction from $2 \mathrm{~L}$ oak chips. Thus, it seems that the extractability of grandinin and roburin $E$ is lower than those of castalagin and vescalagin. This is in accordance with previous results obtained in our laboratory that showed that the lower the surface-to-volume ratio of oak tanks was, the lower proportion of grandinin and roburin $\mathrm{E}$ was found in wines contained in those tanks (data not shown).

2.2.2. Effect of size on the extractability of individual ellagitannins. In order to overlook the influence of toasting, the effect of the size of the oak chips on the extractability of the different ellagitannins has been evaluated from the extraction kinetics of $2 \mathrm{~L}$ and 8L oak chips (see Fig. 1 and Table 2). As a consequence of the difference in the chip width, the surface of $8 \mathrm{~L}$ chips $\left(52 \mathrm{~mm}^{2}\right)$ is almost two-fold bigger than the surface of $2 \mathrm{~L}$ chips $\left(28 \mathrm{~mm}^{2}\right)$. However, as the same amount of oak chips $(4 \mathrm{~g} / \mathrm{L})$ was added to all the model wine solutions, in the case of $2 \mathrm{~L}$ chips there were four times more pieces than in the case of $8 \mathrm{~L}$ chips. Hence, the total surface-tovolume ratio in the solutions containing the smallest oak chips is two-fold greater than that existing in the solutions containing the largest oak chips. As a result, an important reduction in the maximum amount of each ellagitannin extracted from oak chips $\left(C_{\mathrm{eq}}\right)$ can be observed in the $8 \mathrm{~L}$ chips in relation to those observed in the 2L chips. Fig. 1 illustrates this difference in the case of castalagin. However, there were some differences between the different ellagitannins. The value of $C_{\mathrm{eq}}$ for vescalagin extraction was the less

Table 2

Parameters of the Washing $\mathcal{E}$ Two-rates kinetic model for ellagitannin extraction from the different kinds of oak chips

\begin{tabular}{|c|c|c|c|c|c|c|c|c|}
\hline & $C_{\mathrm{eq}}(\mathrm{mg} / \mathrm{L})$ & $F_{\mathrm{w}}$ & $F_{\text {dif }}$ & $F$ & $k_{1}\left(\min ^{-1}\right)$ & $k_{2}\left(\min ^{-1}\right)$ & $R_{\mathrm{adj}}^{2}$ & RMSE \\
\hline \multicolumn{9}{|l|}{$2 \mathrm{~L}$} \\
\hline Castalagin & 21.11 & 0.29 & 0.71 & 0.96 & $1.25 \times 10^{-3}$ & $1.2 \times 10^{-4}$ & 0.997 & 0.19 \\
\hline Vescalagin & 17.71 & 0.31 & 0.69 & 0.89 & $1.76 \times 10^{-3}$ & $1.3 \times 10^{-4}$ & 0.990 & 0.28 \\
\hline Grandinin & 10.28 & 0.27 & 0.73 & 0.74 & $2.06 \times 10^{-3}$ & $4.4 \times 10^{-5}$ & 0.991 & 0.15 \\
\hline Roburin E & 5.38 & 0.21 & 0.79 & 0.62 & $2.22 \times 10^{-3}$ & $6.3 \times 10^{-5}$ & 0.992 & 0.07 \\
\hline \multicolumn{9}{|l|}{$2 \mathrm{M}$} \\
\hline Castalagin & 13.04 & 0.03 & 0.97 & 0.52 & $4.18 \times 10^{-3}$ & $7.2 \times 10^{-4}$ & 0.999 & 0.09 \\
\hline Vescalagin & 9.48 & 0.03 & 0.97 & 0.35 & $2.96 \times 10^{-3}$ & $8.7 \times 10^{-4}$ & 0.991 & 0.22 \\
\hline Grandinin & 2.29 & 0.01 & 0.99 & 0.42 & $1.80 \times 10^{-3}$ & $3.84 \times 10^{-5}$ & 0.995 & 0.02 \\
\hline Roburin E & 1.39 & 0.01 & 0.99 & 0.29 & $3.37 \times 10^{-3}$ & $3.3 \times 10^{-5}$ & 0.991 & 0.01 \\
\hline \multicolumn{9}{|l|}{$8 \mathrm{~L}$} \\
\hline Castalagin & 14.93 & 0.16 & 0.84 & 0.22 & $3.11 \times 10^{-3}$ & $6.5 \times 10^{-4}$ & 0.994 & 0.20 \\
\hline Vescalagin & 13.92 & 0.19 & 0.81 & 0.21 & $4.30 \times 10^{-3}$ & $7.1 \times 10^{-4}$ & 0.995 & 0.29 \\
\hline Grandinin & 6.15 & 0.10 & 0.90 & 0.13 & $5.14 \times 10^{-3}$ & $1.5 \times 10^{-4}$ & 0.991 & 0.13 \\
\hline Roburin E & 2.50 & 0.10 & 0.90 & 0.14 & $5.87 \times 10^{-3}$ & $1.4 \times 10^{-4}$ & 0.991 & 0.05 \\
\hline \multicolumn{9}{|l|}{$8 \mathrm{M}$} \\
\hline Castalagin & 13.90 & 0.04 & 0.96 & 0.42 & $4.97 \times 10^{-3}$ & $7.2 \times 10^{-4}$ & 0.997 & 0.11 \\
\hline Vescalagin & 9.35 & 0.06 & 0.94 & 0.35 & $5.59 \times 10^{-3}$ & $5.56 \times 10^{-4}$ & 0.995 & 0.14 \\
\hline Grandinin & 1.94 & 0 & 1 & 0.22 & $4.90 \times 10^{-3}$ & $1.1 \times 10^{-4}$ & 0.992 & 0.04 \\
\hline Roburin E & 0.96 & 0 & 1 & 0.25 & $6.20 \times 10^{-3}$ & $1.5 \times 10^{-4}$ & 0.998 & 0.01 \\
\hline \multicolumn{9}{|l|}{$8 \mathrm{H}$} \\
\hline Castalagin & 7.13 & 0.01 & 0.99 & 0.35 & $1.07 \times 10^{-2}$ & $1.3 \times 10^{-3}$ & 0.996 & 0.22 \\
\hline Vescalagin & 5.07 & 0.01 & 0.99 & 0.34 & $3.3 \times 10^{-3}$ & $4.5 \times 10^{-4}$ & 0.991 & 0.11 \\
\hline Grandinin & 0.24 & 0 & 1 & 0.38 & $5.28 \times 10^{-3}$ & $9.9 \times 10^{-4}$ & 0.994 & 0.01 \\
\hline Roburin E & 0.29 & 0 & 1 & 0.44 & $7.20 \times 10^{-3}$ & $6.3 \times 10^{-4}$ & 0.991 & 0.01 \\
\hline
\end{tabular}


affected by the reduction of oak chip size, in contrast to the important change of the $C_{\mathrm{eq}}$ value in the case of grandinin and roburin $\mathrm{E}$, thus pointing to the aforementioned differences between the extractability of the different oak ellagitannins. For the same reason, an important reduction in the $F_{\mathrm{w}}$ and, mainly, $F$ values was observed in those containing 8L oak chips in comparison with solutions containing $2 \mathrm{~L}$ oak chips. It was also observed that the lowest reduction of the $F_{\mathrm{w}}$ value with the increase of oak chip size corresponded to the extraction kinetics of vescalagin, thus showing again the higher extractability of this ellagitannin compared to the rest of them.

\subsubsection{Differences on the extractability of individual ellagitannins} depending on the toasting degree of the oak chips. The effect of toasting on the extractability of the different ellagitannins can be compared both in the smallest chips and in the largest ones. In the case of the smallest chips ( $2 \mathrm{~L}$ and $2 \mathrm{M}$, see Table 2 ), toasting can affect almost the entire oak chip, and, as a result, the amounts extracted of ellagitannins were considerably lowered. This is the main reason why extraction from high-toasted oak chips at this size could not be studied. Grandinin and roburin E were the most affected ellagitannins, as can be observed in the important reduction of the $C_{\text {eq }}$ values, whereas castalagin was the less affected. This is in agreement with the lowest thermal resistance of the pentosylated ellagitannins previously pointed out in literature. ${ }^{18}$

The values of $F_{\mathrm{W}}$ close to zero for extraction kinetics from $2 \mathrm{M}$ oak chips can be explained by the almost total thermal degradation of these compounds in the surface of the oak chip. Moreover, in this case, due to the small size of chips, toasting can easily affect the ellagitannins in the outermost layers of the chips, which can explain the lower values of $F$ compared with the kinetics of extraction from 2L oak chips. Moreover, as a consequence of toasting not only the ellagitannin levels of oak wood decrease but also the oak structure undergoes changes, such as the breaking down of cell walls. ${ }^{15}$ This can favor liquid infiltration into the oak chips and, therefore, the extraction of ellagitannins, which might explain the increase of the rate of extraction for the kinetics of castalagin and vescalagin in case of $2 \mathrm{M}$ oak chips compared to $2 \mathrm{~L}$ ones.

Regarding the largest oak chips it was also observed a decrease of $C_{\mathrm{eq}}$ values with toasting, mainly for grandinin and roburin $\mathrm{E}$. However, in this case, toasting would not affect the entire oak chip and the ellagitannins located inside these larger oak chips could resist the thermal treatment, as has previously been pointed out by Jourdes and co-workers. ${ }^{24}$ For this reason, when comparing the extraction models of castalagin and vescalagin of medium-toasted oak chips with those of light-toasted ones, a lower reduction of $C_{\mathrm{eq}}$ values was observed in the case of the biggest chips in relation to the smallest ones. However, when high-toasting conditions were employed in oak cooperage, the thermal degradation of ellagitannins occurs even in the deeper layers of wood. As a consequence, the amounts of grandinin and roburin $\mathrm{E}$ that can be extracted from $8 \mathrm{H}$ oak chips were very low, and an important reduction of the extracted amounts of castalagin and vescalagin was also observed.

On the contrary, an important increase in the $F$ values of the extraction kinetics models from $8 \mathrm{M}$ and $8 \mathrm{H}$ oak chips, compared with those from 8L oak chips, was observed. As explained above, toasting can favor the solvent infiltration into the oak chip. Hence, in the case of $8 \mathrm{M}$ and $8 \mathrm{H}$ oak chips, during the first step of the diffusion process of ellagitannins, solvent may penetrate into deeper layers of the wood, taking place the extraction of a higher proportion of the total ellagitannins that can be extracted from oak chips, thus explaining the higher $F$ values. Moreover, the effect of toasting on wood structure could also explain the increase of the rates of extraction of the diffusion process $\left(k_{1}\right.$ and $\left.k_{2}\right)$. In the case of extraction kinetics models for $8 \mathrm{M}$ and $8 \mathrm{H}$ oak chips it can be observed that, as a consequence of toasting, the first step of the diffusion process is faster compared to that occurring in the models with light-toasted chips, as indicated by the higher values of $k_{1}$, mainly for castalagin (Table 2). However, in the case of extraction from medium-toasted oak chips, the rate of the second step of the diffusion process is hardly affected. Thus, in this case, extraction from the inner layers is not favored by toasting probably due to the fact that toasting at this degree seems not to reach these inner layers. Conversely, in the case of $8 \mathrm{H}$ oak chips, it was observed an important increase of $k_{2}$ values, mainly for grandinin and roburin $\mathrm{E}$, which might indicate that toasting affected greatly the wood structure thus favoring the extraction of these ellagitannins from the inner layers of the oak chips.

\section{Conclusions}

This is the first work that studies the extractability of the main oak ellagitannins from oak chips by means of a kinetic equation. The model here proposed provides better results for explaining the extraction kinetics of the ellagitannins than other models previously used in the literature for explaining the kinetics of solid-toliquid extractions. This model considers an initial extraction (i.e., washing step) followed by a diffusion step, which involves two different processes that follow first-order kinetics at different rates.

Results show important differences on the extractability of the different ellagitannins. Vescalagin shows a higher trend to be released during the washing step than the rest of the ellagitannins, whereas grandinin and roburin E show much lower rates in the diffusion process, which is indicative of a lower extractability of these two ellagitannins. It can also be observed that the increase of the oak chip size implies a reduction in the proportion of ellagitannin released during the fastest steps, although this reduction is less important for vescalagin, thus showing again the higher extractability of this ellagitannin compared to the rest of them.

Toasting causes an important reduction in the amounts of ellagitannins extracted from oak wood. Grandinin and roburin E are the most affected ones whereas castalagin is the less affected. Toasting leads to a drastic reduction in the amounts of ellagitannins released during the washing step. However, it increases the extraction rate during the diffusion step, which indicates that toasting could make the extraction of the ellagitannins easier.

\section{Experimental section}

\subsection{Model wine solutions}

Model wine solutions (MWS) consisted of $12 \%$ ethanol in ultrapure water, $3.8 \mathrm{~g} / \mathrm{L}$ of tartaric acid, and $0.6 \mathrm{~g} / \mathrm{L}$ of potassium chloride (adjusted at pH 3.3 using $\mathrm{NaOH} 1 \mathrm{~N}$ ). Oak chips (4 g/L) were added in the model wine solutions. Two sizes of oak chips were used in the study with different toasting levels for each size. Each type of model wine solution was prepared in triplicate. Oak chips were macerated with orbital agitation for 4 days under an inert atmosphere $\left(\mathrm{N}_{2}\right)$ in order to avoid oxidation, degradation of ellagitannins, and other side-processes, thus enabling the study of kinetics of the single process taking place: the ellagitannin extraction. Sampling was done periodically starting $10 \mathrm{~min}$ after adding the oak chips and samples were analyzed by HPLC-ESI-MS/ MS-multiple reaction monitoring. To avoid losing information about the extraction process, higher number of samples was analyzed in those model wine solutions with the biggest or the more toasted oak chips added. Thus, whereas 12 samples were analyzed in the solutions containing $2 \mathrm{~L}$ oak chips, for better describing the extraction kinetics, 1 and 2 additional samples were analyzed in the case of solutions containing $8 \mathrm{~L}$ oak chips and those containing $2 \mathrm{M}$, $8 \mathrm{M}$ or $8 \mathrm{H}$ oak chips, respectively. 


\subsection{Oak chips}

The oak chips added to model wine solutions were provided from A\&B Group (Brescia, Italy) and were obtained from naturally seasoned (24 months) Q. petraea (Matt.) Liebl oak. Two different sizes (length $\times$ width $\times$ height $=4 \times 2 \times 1 \quad \mathrm{~mm} \quad(2 \mathrm{~mm})$ and $L \times w \times h=4 \times 8 \times 1 \mathrm{~mm}(8 \mathrm{~mm}))$ and three different levels of toasting for each size (light, L; medium, M; and high, $\mathrm{H}$ ) were used. Thus, five kinds of oak chips were tested, two small (2L and $2 \mathrm{M})$ and three large $(8 \mathrm{~L}, 8 \mathrm{M}$ and $8 \mathrm{H}$ ) oak chips. The smallest high-toasted oak chips were not used since previous analysis had shown an important loss of ellagitannins due to toasting leading to very low levels of ellagitannins, mainly of grandinin and roburin E (data not shown).

\subsection{Ellagitannin quantification}

Standards of the main oak ellagitannins analyzed (castalagin, vescalagin, grandinin, and roburin E) were extracted from $Q$. petraea oak chips and purified as it is described by García-Estévez and co-workers. ${ }^{37}$ Castalagin, vescalagin, grandinin, and roburin E were quantified with the previously validated HPLC-ESI-MS/MS-multiple reaction monitoring method. ${ }^{38}$ HPLC analyses were performed in a Hewlett-Packard 1100 series liquid chromatograph (Agilent Technologies, Waldbronn, Germany). MS detection was performed in an API 3200 Qtrap (Applied Biosystems, Darmstadt, Germany) equipped with an ESI source and a triple-quadrupole linear ion trap mass analyzer that was controlled by Analyst 5.1 software (Applied Biosystems, Darmstadt, Germany).

\subsection{Data analysis}

Microsoft Excel 2013 (Redmond, WA, USA) and SOLVER function were used for regression analysis. Experimental data were fitted to different extraction kinetic models (Table 1) by non-linear regression, minimizing the squared errors by using an iteration protocol based on the robust and reliable generalized reduced gradient (GRG) method. The goodness of fit of the models was assessed using adjusted determination coefficient $\left(R_{\mathrm{adj}}^{2}\right)$ and root mean square error (RMSE). Differences on extraction rates of the different ellagitannins due to the kind of oak chips employed were evaluated by comparison of the constants calculated for the best-fitting kinetic model.

\section{Acknowledgements}

Thanks are due to the Spanish MICINN (Project ref. AGL201130254-C02-01) for financial support and to A\&B Group (Brescia, Italy) for chip samples.

\section{Supplementary data}

Supplementary data associated with this article can be found in the online version, at http://dx.doi.org/10.1016/j.tet.2014.10.047.

\section{References and notes}

1. Zamora, F. In Elaboración y crianza del vino tinto: Aspectos científicos y prácticos Ediciones A. Madrid Vicente \& Ediciones Mundi-Prensa: Madrid, Spain, 2003.

2. Puech, J. L.; Feuillat, M.; Boulet, J. C.; Feuillat, F.; Keller, R.; Masson, G.; Moutounet, M.; Naudin, R.; Peyron, D. In Enología: Fundamentos científicos y tecnológicos; Flanzy, C., Ed.; Ediciones A. Madrid Vicente \& Ediciones MundiPrensa: Madrid, Spain, 2003; p 608.

3. Vivas, N.; Glories, Y. Am. J. Enol. Vitic. 1996, 47, 103.

4. Quideau, S.; Jourdes, M.; Saucier, C.; Glories, Y.; Pardon, P.; Baudry, C. Angew. Chem., Int. Ed. 2003, 42, 6012.

5. Quideau, S.; Jourdes, M.; Lefeuvre, D.; Montaudon, D.; Saucier, C.; Glories, Y.; Pardon, P.; Pourquier, P. Chem.-Eur. J. 2005, 11, 6503.

6. Chassaing, S.; Lefeuvre, D.; Jacquet, R.; Jourdes, M.; Ducasse, L.; Galland, S. Grelard, A.; Saucier, C.; Teissedre, P.-L.; Dangles, O.; Quideau, S. Eur. J. Org. Chem. 2010, 2010, 55 .

7. Glabasnia, A.; Hofmann, T. J. Agric. Food Chem. 2006, 54, 3380.

8. Hofmann, T.; Glabasnia, A.; Schwarz, B.: Wisman, K. N.; Gangwer, K. A. Hagerman, A. E. J. Agric. Food Chem. 2006, 54, 9503.

9. Michel, J.; Jourdes, M.; Silva, M. A.; Giordanengo, T.; Mourey, N.; Teissedre, P.-L. J. Agric. Food Chem. 2011, 59, 5677.

10. Chatonnet, P.; Dubourdieu, D. Am. J. Enol. Vitic. 1998, 49, 79.

11. Masson, G.; Moutounet, M.; Puech, J. L. Am. J. Enol. Vitic. 1995, 46, 262.

12. Fernández de Simón, B.; Cadahía, E.; Conde, E.; García-Vallejo, M. C. Holzforschung 1999, 53, 147.

13. Doussot, F.; Pardon, P.; Dedier, J.; De Jéso, B. Analusis 2000, 28, 960.

14. Doussot, F.; De Jéso, B.; Quideau, S.; Pardon, P. J. Agric. Food Chem. 2002, 50 5955.

15. Hale, M. D.; McCafferty, K.; Larmie, E.; Newton, J.; Swan, J. S. Am. J. Enol. Vitic 1999, 50, 495.

16. Matricardi, L.; Waterhouse, A. L. Am. J. Enol. Vitic. 1999, 50, 519.

17. Fernández de Simón, B.; Cadahía, E.; Conde, E.; García-Vallejo, M. C. J. Agric. Food Chem. 1999, 47, 1687

18. Cadahía, E.; Varea, S.; Muñoz, L.; Fernández de Simón, B.; García-Vallejo, M. C. J Agric. Food Chem. 2001, 49, 677.

19. Chira, K.: Teissedre, P. L. Food Chem. 2013, 140, 168

20. Hervé du Penhoat, C.; Michon, V.; Peng, S. Y.; Viriot, C.; Scalbert, A.; Gage, D. J Chem. Soc., Perkin Trans. 1 1991, 1653.

21. Jourdes, M.; Lefeuvre, D.; Quideau, S. In Chemistry and Biology of Ellagitannins. An Underestimated Class of Bioactive Plant Polyphenols; Quideau, S., Ed.; World Scientific: Singapore, 2009; p 320.

22. Quideau, S.; Varadinova, T.; Karagiozova, D.; Jourdes, M.; Pardon, P.; Baudry, C. Genova, P.; Diakov, T.; Petrova, R. Chem. Biodivers. 2004, 1, 247.

23. Fridrich, D.; Glabasnia, A.; Fritz, J.; Esselen, M.; Pahlke, G.; Hofmann, T.; Markor D. J. Agric. Food Chem. 2008, 56, 3010

24. Jourdes, M.; Michel, J.; Saucier, C.; Quideau, S.; Teissedre, P. L. Anal. Bioanal Chem. 2011, 401, 1531.

25. Puech, J. L.; Feuillat, F.; Mosedale, J. R.; Puech, C. Vitis 1996, 35, 211.

26. Karvela, E.; Makris, D. P.; Kefalas, P.; Moutounet, M. Food Chem. 2008, 110, 263.

27. Zanoni, B.; Siliani, S.; Canuti, V.; Rosi, I.; Bertuccioli, M. Int. J. Food Sci. Technol. 2010, 45, 2080

28. Sant'Anna, V.; Brandelli, A.; Ferreira Marczak, L. D.; Tessaro, I. C. Sep. Purif Technol. 2012, 100, 82.

29. Amendola, D.; De Faveri, D. M.; Spigno, G. J. Food Eng. 2010, 97, 384

30. Karacabey, E.; Bayindirli, L.; Artik, N.; Mazza, G. J. Food Process Eng. 2013, 36, 103.

31. Bucic-Kojic, A.; Planinic, M.; Tomas, S.; Bilic, M.; Velic, D. J. Food Process Eng. 2007, 81, 236.

32. Peleg, M. J. Food Sci. 1988, 53, 1216.

33. Kim, J.-W.; Mazza, G. Ind. Eng. Chem. Res. 2007, 46, 7221.

34. Cacace, J. E.; Mazza, G. J. Food Process Eng. 2003, 59, 379.

35. Simeonov, E.; Tsibranska, I.; Minchev, A. Chem. Eng. J. 1999, 73, 255.

36. Simeonov, E.; Koleva, V. Chem. Biochem. Eng. Q. 2012, 26, 249.

37. García-Estévez, I.; Escribano-Bailón, M. T.; Rivas-Gonzalo, J. C.; Alcalde-Eon, C. Anal. Chim. Acta 2010, 660, 171.

38. García-Estévez, I.; Escribano-Bailón, M. T.; Rivas-Gonzalo, J. C.; Alcalde-Eon, C. J Agric. Food Chem. 2012, 60, 1373. 